\title{
Face-name learning in older adults: a benefit of hyper-binding
}

\author{
Jennifer C. Weeks ${ }^{1,2}$ • Renée K. Biss ${ }^{3}$ - Kelly J. Murphy ${ }^{2,3} \cdot$ Lynn Hasher $^{1,2}$
}

Published online: 1 February 2016

(C) Psychonomic Society, Inc. 2016

\begin{abstract}
Difficulty remembering faces and corresponding names is a hallmark of cognitive aging, as is increased susceptibility to distraction. Given evidence that older adults spontaneously encode relationships between target pictures and simultaneously occurring distractors (a hyper-binding phenomenon), we asked whether memory for face-name pairs could be improved through prior exposure to faces presented with distractor names. In three experiments, young and older adults performed a selective attention task on faces while ignoring superimposed names. After a delay, they learned and were tested on face-name pairs that were either maintained or rearranged from the initial task but were not told of the connection between tasks. In each experiment, older but not younger participants showed better memory for maintained than for rearranged pairs, indicating that older adults' natural propensity to tacitly encode and bind relevant and irrelevant information can be employed to aid face-name memory performance.
\end{abstract}

Keywords Aging \& attention · Aging \& memory $\cdot$ Implicit memory $\cdot$ Inhibition

Jennifer C. Weeks

jennifer.weeks@utoronto.ca

$\triangle$ Lynn Hasher

hasher@psych.utoronto.ca

1 Rotman Research Institute, Baycrest, Toronto, Ontario, Canada M6A 2E1

2 Department of Psychology, University of Toronto, 100 St. George Street, Toronto, Ontario M5S 3G3, Canada

3 Neuropsychology \& Cognitive Health, Baycrest, Toronto, Ontario, Canada M6A 2E1
Age-related deficits are seen across a range of intentional memory tasks (e.g., Balota, Dolan, \& Duchek, 2000), including a disproportionate impairment in learning face-name associations (e.g., Crook \& West, 1990). In contrast, implicit (or unintentional) memory is relatively preserved in old age (Fleischman, Wilson, Gabrieli, Bienias, \& Bennett, 2004), and previous work has shown that aging may actually increase implicit memory for distracting items that appear outside of the focus of attention (e.g., Campbell, Grady, Ng, \& Hasher, 2012; Rowe, Valderrama, Hasher, \& Lenartowicz, 2006). The observed age-related increase in implicit memory for distractors has been attributed to attentional dysregulation at encoding in older adulthood (Hasher, Zacks, \& May, 1999; Rowe et al., 2006).

Although attentional dysregulation can have undesirable effects on older adults' cognitive performance (Weeks \& Hasher, 2014), the presence of distraction can also be beneficial when the distractors subsequently reappear as to-beremembered items in a later memory task (e.g., Thomas \& Hasher, 2012) and when distraction serves as a rehearsal opportunity between initial learning and final recall (Biss, Ngo, Hasher, Campbell, \& Rowe, 2013). These effects appear to be implicit, as individuals report having neither consciously attended to distractors nor awareness of the connection between tasks.

Older adults also show evidence of forming spontaneous associations between cooccurring targets and distractors, or hyper-binding (Campbell, Hasher, \& Thomas, 2010). This effect was demonstrated in a task in which older and younger participants first performed a one-back identity judgment task on pictures, ignoring superimposed words. After a delay, they studied a series of picture-word pairs, some of which were previously presented in the one-back task (maintained), and others which were rearranged from previously seen stimuli (disrupted). Older but not younger adults showed better memory for maintained than disrupted pairs, despite being unaware 
of the repetition of words between tasks. Thus, older adults retain not only item-level information about prior distraction but also information about cooccurrences of target and nontarget stimuli, and this implicit knowledge can influence subsequent explicit memory performance.

In three experiments reported here, we asked whether the hyper-binding paradigm could be used as a device for helping older adults learn face-name pairings, a task that is known to become increasingly difficult in older age (e.g. Crook \& West, 1990).

\section{Experiment 1a}

Younger and older adults first performed a selective attention task in which they attended to faces and ignored superimposed names. After a delay, they studied a series of face-name pairs and then performed a cued recall test in which faces were presented and names were to be recalled. All faces and names had been seen on the one-back task, but half of the pairs on the study list were identical to those seen initially (maintained), and the other half of pairs were rearranged (disrupted). Participants were not informed of the relationship between the first and final tasks. Cued recall was compared for maintained and disrupted pairs, with the assumption that any observed difference would be the product of binding distracting names to faces in the initial task and tacit transfer of that knowledge to the final test task.

\section{Method}

\section{Participants}

Twenty young adults, ages 16 to 21 years ( 14 women), and 20 older adults, ages 61 to 75 years ( 16 women), were tested (see
Table 1 for demographics for all experiments). Sample sizes were based on those used by Campbell et al. (2010). Young adults were undergraduates and received partial course credit for their participation. Older adults were volunteers from the community and received monetary compensation. No participants reported a history of psychiatric or neurological illness. Given our interest in tacit transfer, one young and one older adult were replaced prior to data analysis because they reported being aware of the connection between tasks. None of the participants in any of the experiments had previously participated in a study measuring implicit effects of distraction.

Older adults were significantly more educated than the young adults, who were currently enrolled in an undergraduate program, $t(38)=6.34, p<.001, d=2.42$, and scored higher on the Shipley (1946) vocabulary test compared to younger adults, $t(38)=7.23, p<.001, d=2.25$.

\section{Materials}

Thirty-four young adult (ages 18-30) and 34 older adult faces (ages 60-94) were selected from the Centre for Lifespan Longevity Face Database (Minear \& Park, 2004). Within each age category, two counterbalanced lists of eight faces $(50 \%$ female) were compiled and matched on perceived age, familiarity, mood, memorability, and picture quality (Kennedy, Hope, \& Raz, 2009). Eighteen filler faces were chosen from each age category in order to obscure the connection between the one-back and cued recall tasks. All images were in grayscale and pictured people from the shoulders up.

An equal number of first names were chosen from the top 50 to 100 baby names for the appropriate birth years (http:// www.ssa.gov/OACT/babynames). Because of the welldocumented same-age bias in remembering faces (e.g., Wiese, Komes, \& Schweinberger, 2013), participants only viewed faces from their own age category. Older and younger
Table 1 Demographic and oneback Accuracy Scores

\begin{tabular}{|c|c|c|c|c|}
\hline Group & Age & Education & Vocabulary & One-back accuracy \\
\hline \multicolumn{5}{|l|}{ Experiment 1a } \\
\hline Younger adults & $18.4(1.3)$ & $12.8(1.8)$ & $30.6(3.8)$ & $97 \%(7 \%)$ \\
\hline Older adults & $69.9(4.3)$ & $17.8(2.3)$ & $37.6(2.2)$ & $77 \%(21 \%)$ \\
\hline \multicolumn{5}{|l|}{ Experiment $1 \mathrm{~b}$} \\
\hline Younger adults & $18.9(0.9)$ & $13.3(1.0)$ & $27.7(4.4)$ & - \\
\hline Older adults & $69.6(5.1)$ & $15.8(3.3)$ & $32.8(5.4)$ & - \\
\hline \multicolumn{5}{|l|}{ Experiment 2} \\
\hline Younger adults & $19.5(3.4)$ & $13.1(1.9)$ & $29.6(4.0)$ & $95 \%(12 \%)$ \\
\hline Older adults & $67.1(4.0)$ & $17.0(2.8)$ & $35.4(4.5)$ & $77 \%(20 \%)$ \\
\hline \multicolumn{5}{|l|}{ Experiment 3} \\
\hline Younger adults & $19.7(2.5)$ & $13.7(1.8)$ & $29.8(2.7)$ & $88 \%(16 \%)$ \\
\hline Older adults & $67.9(5.0)$ & $18.2(5.2)$ & $34.7(3.3)$ & $86 \%(15 \%)$ \\
\hline
\end{tabular}

Note. Standard deviations are in parentheses. One-back accuracy calculated as a percentage of hits minus false alarms. 
face sets were also matched on familiarity, mood, memorability, and picture quality.

For each age group, critical faces were pseudorandomly matched with sex- and age-appropriate names to form two sets of 16 critical face-name pairs (eight maintained and eight disrupted) for the cued recall task. Pair type was counterbalanced across participants. The remainder of the faces and names were pseudorandomly combined and used as filler pairs in the oneback task.

\section{Procedure}

Participants were told that the experiment consisted of four unrelated tasks. First, they performed a one-back task on faces, pressing the spacebar when a face repeated, while ignoring superimposed names. Stimuli were presented for $1,000 \mathrm{~ms}$ each, with a $500 \mathrm{~ms}$ interstimulus interval. The task began with five faces with no superimposed text, followed by six filler faces with superimposed filler names, followed by 90 trials that either contained filler face-name pairs (42 trials in total; each face and name presented three times each but never in the same combination) or critical face-name pairs (48 trials each; each critical face-name pair presented together three times). Finally, six filler pairs served as a recency buffer. Targets on the one-back task appeared every six trials on average and never contained a critical, to-be-tested pair.

During a 10-minute delay interval, participants completed two nonverbal visuospatial detection tasks. Participants were then asked to imagine that they were invited to a party at which they would meet many interesting guests, whose faces and names they were going to learn beforehand. They then studied eight maintained and eight disrupted face-name pairs presented in a random order for 4,000 ms each. Names were presented superimposed on the forehead, as in the one-back task. Immediately after study, they were shown the faces in a different random order and were asked to say the corresponding name. A graded awareness questionnaire was then administered to determine whether participants recognized the studied pairs as having appeared in the one-back task.

\section{Results}

Accuracy on the one-back task was calculated as the percentage of hits minus false alarms (see Table 1). As in previous studies, young adults were more accurate than older adults in detecting repetitions, $t(38)=4.02, p<.001, d=1.28$, (e.g., Campbell et al., 2010).

Cued recall performance on the face-name learning task was entered into an Age (younger, older) $\times$ Pair Type (maintained, disrupted) mixed ANOVA. Young adults remembered more names than older adults, as seen in Fig. 1a, $F(1,38)=$ $18.47, p<.001, \eta_{\mathrm{p}}{ }^{2}=.33$.
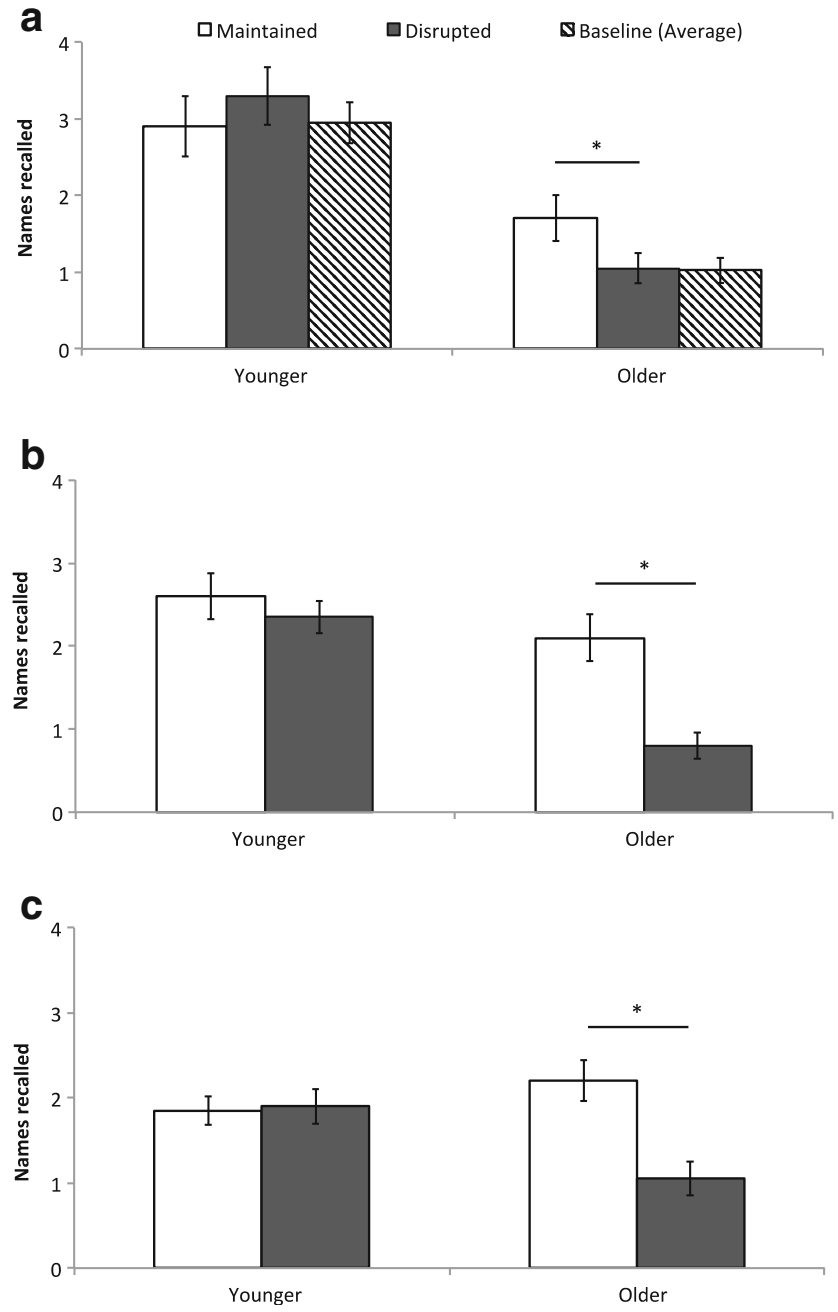

Fig. 1 Mean number of names correctly recalled in cued recall task for three experiments. Error bars represent one standard error of the mean. In (a) Experiment 1, there were eight maintained and eight disrupted sameage face-name pairs. Older but not younger adults showed better memory for maintained pairs from the one-back task compared to disrupted pairs as well as to a control group that did not perform the one-back task (shown here collapsed across a dummy variable). In (b) Experiment 2, there were four maintained and four disrupted same-age face-name pairs, and older adults again showed a benefit for maintained pairs, eliminating the age difference in the maintained condition. In (c) Experiment 3, the effects from Experiment 2 were replicated with opposite-age pairs (four maintained, four disrupted).

The interaction was also significant, $F(1,38)=5.42, p=$ $.025, \eta_{\mathrm{p}}{ }^{2}=.13$, such that older adults had significantly better memory for maintained than disrupted pairs $(p=.049)$ but young adults showed no influence of pair type $(p=.218)$.

\section{Experiment 1b}

Older adults' cued recall performance was implicitly influenced by prior exposure to items, but it remains unclear whether this effect is primarily driven by an increase in 
memory for maintained pairs or a decrease in memory for disrupted pairs due to interference (Biss, Campbell, \& Hasher, 2013). To disentangle these effects, we tested a group of naïve participants on only the face-name paired associates task to obtain a baseline level of performance for people with no prior exposure to face or name stimuli.

\section{Method}

Twenty young adults, ages 17 to 21 years ( 17 women), and 20 older adults, ages 60 to 79 years (12 women), performed the face-name learning task after participating in an unrelated psychology study. These participants were comparable to those in Experiment 1a in terms of age, $t<1$, and education, $t(78)=1.36, p=.177$ (see Table 1). The sole change from the previous experiment was the elimination of the one-back and filler tasks.

\section{Results}

Since participants in this control group did not perform the one-back task, pair type was coded as a dummy variable, and the data were analyzed using an Experiment $(1 \mathrm{a}, 1 \mathrm{~b}) \times$ Age (younger, older) $\times$ Pair Type (maintained, disrupted) mixed ANOVA. The three-way interaction was significant, $F(1,76)$ $=12.69, p=.001, \eta_{\mathrm{p}}{ }^{2}=.14$, and the two-way Age $\times$ Experiment interaction was only significant for maintained pairs, $F(1,76)=4.72, p=.033, \eta_{\mathrm{p}}{ }^{2}=.06$. Post hoc pairwise comparisons indicated that the source of the interaction was better memory for maintained pairs in primed compared to control older adults $(p=.003)$, and no such difference among younger adults $(p>.250)$. As expected, the control group showed no effect of the dummy variable, $t<1$, so baseline scores are presented as averages in Fig. 1a. The data suggest that older adults experience a benefit for maintained pairs without a cost for disrupted pairs (but see Campbell et al., 2010, Experiment 1). However, overall performance was very low (i.e., participants in the control condition correctly remembered only one pair on average), so there may be insufficient range to detect a difference between disrupted and baseline conditions, especially with a between-groups comparison. A within-groups comparison would be more powerful but was not included here to avoid lengthening the to-belearned list and potentially reducing older adults' performance to floor levels.

\section{Experiment 2}

Although older adults' face-name memory was improved in Experiment 1 by previous exposure to relevant distraction, younger adults still showed a substantial overall memory advantage (see Fig. 1a). Given that age-related impairments in memory are exacerbated by long study lists (e.g., Crook \& West, 1990), we considered the possibility that older adults' performance on our task may be further improved if there were fewer pairs to study, and therefore less interference between pairs. We hypothesized that reducing the number of pairs would not affect younger adults' performance, since older adults are generally more vulnerable to list length effects than are young adults (e.g., Cohen, Sandler, \& Schroeder, 1987; Crook \& West, 1990). To test this prediction, we replicated Experiment 1a using only eight to-be-remembered pairs (four maintained, four disrupted) and a proportionately shortened version of the one-back task.

\section{Method}

\section{Participants}

Twenty young adults, ages 18 to 29 years (14 women), and 20 older adults, ages 60 to 76 years ( 12 women), were recruited as before. As in Experiment 1, older adults were more educated, $t(38)=5.22, p<.001, d=1.63$, and had higher vocabulary scores, $t(36)=4.22, p<.001, d=1.36$ (data missing from two individuals), compared to young adults. Prior to data analysis, one younger and one older adult were replaced for being aware of the connection between tasks.

\section{Materials \& procedure}

Two counterbalanced lists of four critical face-name pairs were created for each age category, as described in Experiment 1. In order to maintain a ratio of critical to filler items similar to Experiment 1, eight filler faces and names were selected for use in the one-back task. The one-back task began with three filler trials, followed by 42 trials that either contained a critical pair (three repetitions each for a total of 24 trials) or a filler pair (18 trials), followed by a recency buffer of three filler trials. After a 10-minute delay, participants studied the eight face-name pairs and were given an immediate cued recall test.

\section{Results}

Young adults again outperformed older adults on the one-back task, $t(37)=3.38, p=.002, d=1.09$ (data missing from one older adult due to program malfunction; see Table 1). An Age (younger, older) $\times$ Pair Type (maintained, disrupted) mixed ANOVA on cued recall data resulted in main effects of Age, $F(1,38)=16.85, p<.001, \eta_{\mathrm{p}}{ }^{2}=.31$, and Pair Type, $F(1,38)=$ $13.05, p=.001, \eta_{\mathrm{p}}{ }^{2}=.26$, which were qualified by a reliable interaction, $F(1,38)=5.99, p=.019, \eta_{\mathrm{p}}^{2}=.14$ (see Fig. 1b). Post hoc pairwise comparisons revealed that older adults again had better memory for maintained than disrupted pairs $(p<$ $.001)$, while young adults showed no such difference $(p>$ 
.250). Older adults recalled fewer names from disrupted pairs than did younger adults $(p<.001)$, but the age difference on maintained pairs was no longer significant $(p=.211)$. Evidently, older adults were able to perform at the level of young adults under circumstances in which interference is minimized and distraction plays a supportive role.

\section{Experiment 3}

Much work has been done examining the same-age bias in face memory, which is reliable in young adults (Anastasi \& Rhodes, 2006) but somewhat less so for older adults (Fulton \& Bartlett, 1991). In this third experiment, our goal was to determine whether the beneficial effects of preexposure to distraction can extend to the learning of opposite-age faces.

\section{Method}

\section{Participants}

Samples of 20 young adults, ages 18 to 27 years ( 17 women), and 20 older adults, ages 61 to 79 years (16 women), were recruited as before. Again, older participants had more years of education, $t(37)=3.70, p=.001, d=1.16$ (data missing from one individual), and higher vocabulary scores, $t(38)=$ $5.12, p<.001, d=1.63$, than young adults.

\section{Materials \& procedure}

Experiment 3 was identical to Experiment 2, except that young participants performed the one-back and cued recall tasks on the older faces, and older participants performed the tasks on the younger faces.

\section{Results}

Unlike in Experiments 1 and 2, there was no age difference in accuracy on the one-back test, $t<1$. Accuracy was compared across Experiments 2 and 3 using participant age group as one factor and age group of faces as another. There was a main effect of Participant Age, $F(1,75)=7.60, p=.007, \eta_{\mathrm{p}}{ }^{2}=.09$, showing better one-back performance in young compared to older adults, and a main effect of Face Age, $F(1,75)=4.91, p$ $=.030, \eta_{\mathrm{p}}{ }^{2}=.06$, showing that both younger and older adults performed better on the one-back task with young faces compared to older faces, perhaps owing to older adults' extensive experience and familiarity with young faces (Wiese et al., 2013).

Hyper-binding was tested with an Age (younger, older) $\times$ Pair Type (maintained, disrupted) mixed ANOVA on cued recall (see Fig. 1c). The main effect of Pair Type, $F(1,38)=$ $23.58, p<.001, \eta_{\mathrm{p}}{ }^{2}=.38$, again was qualified by a significant interaction, $F(1,38)=28.06, p<.001, \eta_{\mathrm{p}}^{2}=.43$, with older adults showing a benefit for maintained pairs $(p<.001)$ and young adults showing no difference $(p>.250)$. As in Experiment 2, age differences were present in the disrupted condition $(p=.005)$ but were eliminated in the maintained condition $(p=.233)$. Thus, the benefit of exposure to previous distraction extends to opposite-age faces, at least for older adults.

To examine the existence of a same-age bias, we compared total recall across age groups in Experiments 2 and 3. As expected, young adults outperformed older adults, $F(1,76)$ $=12.34, p=.001, \eta_{\mathrm{p}}{ }^{2}=.14$, and the Age $\times$ Experiment interaction was also significant, $F(1,76)=4.56, p=.036, \eta_{\mathrm{p}}{ }^{2}=$ .06 . Only young adults showed a decrease in memory performance for opposite-age pairs $(\mathrm{p}=.028)$ while older adults showed equivalent memory performance for same- and opposite-age pairs $(p>.250)$. This result is consistent with previous findings of larger same-age effects in face memory in young adults compared with older adults (e.g., Bryce \& Dodson, 2013).

\section{Discussion}

In three experiments, we demonstrated a method to boost older (but not younger) adults' memory for face-name pairs. We did this by capitalizing on the hyper-binding effect (Campbell et al., 2010), which describes older adults' tendency to automatically form associations between targets and cooccurring distractors, and transfer this knowledge to a memory task. This evidence that older adults encode and bind distractor names to target faces is particularly dramatic given that faces are powerful stimuli believed to automatically capture attention (e.g., Sato \& Kawahara, 2014).

Although we are reasonably certain that the transfer of distraction was implicit given that no participants reported being aware of the underlying connection between tasks, we recognize the shortcomings of self-report measures and therefore do not rule out the possibility of contamination by explicit memory. However, our findings are in line with classic literature demonstrating that explicit tasks can be influenced by implicit memory (e.g., Jacoby, 1991), and this influence is often greater for older adults, who are more likely to commit memory errors reflective of a reliance on familiarity (Jennings \& Jacoby, 1993). Relying on implicit memory during retrieval may even be adaptive for older adults, who have also been shown to incidentally encode more information about the environment than do young adults (Campbell et al., 2010; Campbell, Zimerman, Healey, Lee, \& Hasher, 2012). Neuroimaging data corroborate the idea that older adults unintentionally monitor nontarget stimuli in the environment (Campbell, Grady, et al., 2012). Overall, our findings reinforce Craik's (1986) notion that implicit environmental 
support plays a large role in older adults' explicit memory and raise the possibility that the environment can be manipulated to optimize older adults' performance, specifically by minimizing harmful interference and maximizing helpful distraction.

Our data provide evidence for two seemingly contradictory ideas in the aging literature: hyper-binding (Campbell et al., 2010), and the age-related deficit in associative binding (e.g., Naveh-Benjamin, 2000). In Experiment 1a, we observed the typical age-related impairment in explicit memory for pairs, but the benefit for maintained pairs seen in all three older adult samples suggests that this impairment can be offset by previous exposure to helpful distraction. Other work has demonstrated that age differences in associative memory are eliminated when the test task is implicit rather than explicit (Dew \& Giovanello, 2010), which, along with growing evidence for hyper-binding (e.g. Campbell et al., 2010), suggests that older adults' impairment lies less in forming associations and more in retrieving them.

The present findings may also have clinical relevance because declining face-name memory is not only a hallmark of aging (e.g., Crook \& West, 1990) but can also be a symptom of mild cognitive impairment (Hampstead, Sathian, Moore, Nalisnick, \& Stringer, 2008; Troyer et al., 2012) and progression to Alzheimer's disease (Werheid \& Clare, 2007). To date, most interventions aimed at improving face-name memory in older adults have focused on training explicit memory strategies (Hampstead et al., 2008) and imagery (Belleville et al., 2006). Extrapolating from the current results, future interventions may capitalize on older adults' natural tendency to tacitly rely on incidentally encoded information at retrieval (Biss, Ngo, et al., 2013).

Author Note The authors gratefully acknowledge the assistance of lab manager Elizabeth Howard as well as undergraduate students Kyle Hamel and Stephen Cooper in data collection. This work was funded by National Science and Engineering Council of Canada (NSERC) grant 487235 to L. Hasher, an Alzheimer Society of Canada Postdoctoral Award to R. K. Biss, and an NSERC Canada Graduate Scholarship (Doctoral) to J. C. Weeks.

\section{References}

Anastasi, J. S., \& Rhodes, M. G. (2006). Evidence for an own-age bias in face recognition. North American Journal of Psychology, 8(2), 237252.

Balota, D. A., Dolan, P. O., \& Duchek, J. M. (2000). Memory changes in healthy older adults. In E. Tulving \& F. I. M. Craik (Eds.), The Oxford handbook of memory (pp. 395-409). New York, NY: Oxford University Press.

Belleville, S., Gilbert, B., Fontaine, F., Gagnon, L., Menard, E., \& Gauthier, S. (2006). Improvement of episodic memory in persons with mild cognitive impairment and healthy older adults: Evidence from a cognitive intervention program. Dementia and Geriatric Cognitive Disorders, 22, 486-499.
Biss, R. K., Campbell, K. L., \& Hasher, L. (2013a). Interference from previous distraction disrupts older adults' memory. Journals of Gerontology. Series B, Psychological Sciences and Social Sciences, 68, 558-561.

Biss, R. K., Ngo, K. W. J., Hasher, L., Campbell, K. L., \& Rowe, G. (2013b). Distraction can reduce age-related forgetting. Psychological Science, 24, 448-455.

Bryce, M. S., \& Dodson, C. S. (2013). Cross-age effect in recognition performance and memory monitoring for faces. Psychology and Aging, 28(1), 87-98.

Campbell, K. L., Grady, C. L., Ng, C., \& Hasher, L. (2012a). Age differences in the frontoparietal cognitive control network: Implications for distractibility. Neuropsychologia, 50(9), 2212-2223.

Campbell, K. L., Hasher, L., \& Thomas, R. C. (2010). Hyper-binding: A unique age effect. Psychological Science, 21(3), 399-405.

Campbell, K. L., Zimerman, S., Healey, M. K., Lee, M., \& Hasher, L. (2012b). Age differences in visual statistical learning. Psychology and Aging, 27(3), 650-656.

Cohen, R. L., Sandler, S. P., \& Schroeder, K. (1987). Aging and memory for words and action events: Effects of item repetition and list length. Psychology and Aging, 2(3), 280-285.

Craik, F. I. (1986). A functional account of age differences in memory. In F. Klix \& H. Hagendorf (Eds.), Human memory and cognitive capabilities: Mechanisms and performances (pp. 409-422). Amsterdam, The Netherlands: Elsevier.

Crook, T. H., \& West, R. L. (1990). Name recall performance across the adult life-span. British Journal of Psychology, 81(3), 335-349.

Dew, I. T., \& Giovanello, K. S. (2010). Differential age effects for implicit and explicit conceptual associative memory. Psychology and Aging, 25(4), 911-921.

Fleischman, D. A., Wilson, R. S., Gabrieli, J. D., Bienias, J. L., \& Bennett, D. A. (2004). A longitudinal study of implicit and explicit memory in old persons. Psychology and Aging, 19(4), 617-625.

Fulton, A., \& Bartlett, J. C. (1991). Young and old faces in young and old heads: The factor of age in face recognition. Psychology and Aging, 6, 623-630.

Hampstead, B. M., Sathian, K., Moore, A. B., Nalisnick, C., \& Stringer, A. Y. (2008). Explicit memory training leads to improved memory for face-name pairs in patients with mild cognitive impairment: Results of a pilot investigation. Journal of the International Neuropsychological Society, 14(05), 883-889.

Hasher, L., Zacks, R. T., \& May, C. P. (1999). Inhibitory control, circadian arousal, and age. In D. Gopher \& A. Koriat (Eds.), Attention \& performance, XVII: Cognitive regulation of performance: Interaction of theory and application (pp. 653-675). Cambridge, MA: MIT Press.

Jacoby, L. L. (1991). A process dissociation framework: Separating automatic from intentional uses of memory. Journal of Memory and Language, 30, 513-541.

Jennings, J. M., \& Jacoby, L. L. (1993). Automatic versus intentional uses of memory: Aging, attention, and control. Psychology and Aging, 8(2), 283-293.

Kennedy, K. M., Hope, K., \& Raz, N. (2009). Life span adult faces: Norms for age, familiarity, memorability, mood, and picture quality. Experimental Aging Research, 35(2), 268-275.

Minear, M., \& Park, D. C. (2004). A lifespan database of adult facial stimuli. Behavior Research Methods, Instruments, \& Computers, 36(4), 630-633.

Naveh-Benjamin, M. (2000). Adult age differences in memory performance: Tests of an associative deficit hypothesis. Journal of Experimental Psychology Learning Memory and Cognition, 26(5), $1170-1187$.

Rowe, G., Valderrama, S., Hasher, L., \& Lenartowicz, A. (2006). Attentional disregulation: A benefit for implicit memory. Psychology and Aging, 21(4), 826-830. 
Sato, S., \& Kawahara, J. (2014). Task-irrelevant faces capture attention regardless of perceptual load. Journal of Vision, 14(10), 319.

Shipley, W. C. (1946). Institute of living scale. Los Angeles, CA: Western Psychological Services.

Thomas, R. C., \& Hasher, L. (2012). Reflections of distraction in memory: Transfer of previous distraction improves recall in younger and older adults. Journal of Experimental Psychology Learning Memory and Cognition, 38, 30-39.

Troyer, A., Murphy, K. J., Anderson, N., Craik, F. I. M., Moscovitch, M., Maoine, A., \& Fuqiang, G. (2012). Impaired associative but not item memory in mild cognitive impairment: Relationship to hippocampal volume and apolipoprotien E. Neuropsychologia, 50, 3721-3728.
Weeks, J. C., \& Hasher, L. (2014). The disruptive - and beneficialeffects of distraction on older adults' cognitive performance. Frontiers in Psychology, 5, 133. doi:10.3389/fpsyg.2014.00133

Werheid, K., \& Clare, L. (2007). Are faces special in Alzheimer's disease? Cognitive conceptualisation, neural correlates, and diagnostic relevance of impaired memory for faces and names. Cortex, 43(7), 898-906.

Wiese, H., Komes, J., \& Schweinberger, S. R. (2013). Ageing faces in ageing minds: A review on the own-age bias in face recognition. Visual Cognition, 21(9/10), 1337-1363. 